Hautarzt 2020 $71: 898$

https://doi.org/10.1007/s00105-020-04711-2

Online publiziert: 19 . Oktober 2020

(c) Springer Medizin Verlag $\mathrm{GmbH}$, ein Teil von Springer Nature 2020

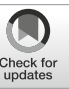

H. Schöfer ${ }^{1} \cdot$ M. Enders ${ }^{2} \cdot$ S. Esser ${ }^{3} \cdot$ C. Feiterna-Sperling ${ }^{4} \cdot$ H.-J. Hagedorn ${ }^{5} \cdot$

G. Magistro ${ }^{6} \cdot$ C. Mayr ${ }^{7} \cdot$ D. Münstermann ${ }^{5} \cdot K$. Hahn $^{8} \cdot$ K. Jansen ${ }^{9} \cdot$ M. Klein ${ }^{10}$. W. Krause ${ }^{11} \cdot$ M. Maschke $^{12} \cdot$ F. R. Ochsendorf ${ }^{13} \cdot$ S. Osowski ${ }^{14} \cdot$ K. U. Petry ${ }^{15}$.

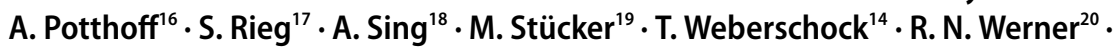
N. H. Brockmeyer ${ }^{16}$

' Deutsche Klinik für Diagnostik, Helios Dr. Horst Schmidt Kliniken, Wiesbaden, Deutschland; ${ }^{2}$ Labor Prof. Gisela Enders \& Kollegen MVZ Stuttgart, Stuttgart, Deutschland; ${ }^{3}$ Leiter der HIV/STD-Ambulanz, Klinik für Dermatologie und Venerologie, Universitätsklinikum Essen, Essen, Deutschland; ${ }^{4}$ Klinik für Pädiatrie $\mathrm{m} . \mathrm{S}$ Pneumologie, Immunologie und Intensivmedizin, Charité Universitätsmedizin Berlin, Berlin, Deutschland; ${ }^{5}$ MVZ Labor Krone, Bad Salzuflen, Deutschland; ${ }^{6}$ Urologische Klinik, Ludwig-Maximilians-Universität München, München, Deutschland; ' ${ }^{7}$ Hausärztliche Betreuung, Zentrum für Infektiologie Berlin, Prenzlauer Berg (ZIBP), MVZ, Berlin, Deutschland; ${ }^{8}$ Klinik für Neurologie, Universitätsmedizin Charité, Campus Charité Mitte, Berlin, Deutschland; ${ }^{9}$ Abteilung für Infektionsepidemiologie, Fachgebiet für HIV/AIDS und andere sexuell oder durch Blut übertragbare Infektionen, Robert Koch-Institut, Berlin, Deutschland; ${ }^{10}$ Abteilung: Neurologische Klinik, Ludwig-Maximilians-Universität München, Klinikum Großhadern, München, Deutschland; "Hautklinik der Philipps-Universität, Marburg, Deutschland; ${ }^{12}$ Abteilung: Neurologie, Neurophysiologie und neurologische Frührehabilitation, MVZ der Barmherzigen Brüder Trier, Sektion Neurologie, Psychiatrie, Trier, Deutschland; ${ }^{13} \mathrm{Klinik}$ für Dermatologie, Venerologie und Allergologie (KDVA), Universitätsklinikum Frankfurt, Frankfurt, Deutschland; ${ }^{14} \mathrm{Klinik}$ f. Dermatologie, Venerologie und Allergologie (KDVA) und Evidenzbasierte Medizin, Institut für Allgemeinmedizin, GoetheUniversität Frankfurt, Frankfurt, Deutschland; ${ }^{15}$ Frauenklinik, Klinikum der Stadt Wolfsburg, Wolfsburg, Deutschland; ${ }^{16}$ WIR "Walk In Ruhr" im St. Elisabeth-Hospital, Bochum, Deutschland; ${ }^{17}$ Abteilung Infektiologie, Klinik für Innere Medizin II, Universitätsklinikum Freiburg, Freiburg, Deutschland; ${ }^{18}$ Bayerisches Landesamt für Gesundheit und Lebensmittelsicherheit (LGL), Oberschleißheim, Deutschland; ${ }^{19} \mathrm{Klinik}$ für Dermatologie, Venerologie und Allergologie Ruhr-Universität Bochum, Bochum, Deutschland; ${ }^{20}$ Klinik für Dermatologie, Venerologie und Allergologie, Division of Evidence-Based Medicine (dEBM), Charité - Universitätsmedizin Berlin, Berlin, Deutschland

\title{
Erratum zu: Diagnostik und Therapie der Syphilis. Aktualisierung der S2k-Leitlinie 2020 der Deutsche STI- Gesellschaft (DSTIG) in Kooperation mit folgenden Fachgesellschaften: DAIG, dagnä, DDG, DGA, DGGG, DGHM, DGI, DGN, DGPI, DGU, RKI
}

In der ersten Onlineversion dieses Beitrags waren 2 Autorennamen falsch geschrieben, bei einem Autor war die Institutsangabe falsch. Wir bitten um Kenntnisnahme.

Der Originalbeitrag wurde korrigiert.

\section{Korrespondenzadresse}

Prof. Dr. med. H. Schöfer

Deutsche Klinik für Diagnostik, Helios Dr. Horst Schmidt Kliniken

Aukamm-Allee 33, 65191 Wiesbaden,

Deutschland

helmutschoefer.ni@gmail.com

e-Version des Originalartikels ist unte zufinden. 